\title{
PRRT2 mutations lead to neuronal dysfunction and neurodevelopmental defects
}

\author{
Yo-Tsen Liu',2, Fang-Shin Nian ${ }^{3,4}$, Wan-Ju Chou ${ }^{4}$, Chin-Yin Tai ${ }^{5}$, Shang-Yeong \\ Kwan',2, Chien Chen', ${ }^{1,2}$, Pei-Wen Kuo ${ }^{4}$, Po-Hsi Lin' ${ }^{2}$, Chin-Yi Chen ${ }^{6}$, Chia-Wei Huang ${ }^{4}$, \\ Yi-Chung Lee ${ }^{1,2,7}$, Bing-Wen Soong ${ }^{1,2,7}$ and Jin-Wu Tsai ${ }^{4,7,8}$ \\ ${ }^{1}$ Department of Neurology, Neurological Institute, Taipei Veterans General Hospital, Taipei, Taiwan \\ 2 Department of Neurology, National Yang-Ming University School of Medicine, Taipei, Taiwan \\ 3 Program in Molecular Medicine, National Yang-Ming University and Academia Sinica, Taipei, Taiwan \\ ${ }^{4}$ Institute of Brain Science, National Yang-Ming University, Taipei, Taiwan \\ ${ }^{5}$ Istitute of Pharmaceutics, Development Center for Biotechnology, New Taipei City, Taiwan \\ ${ }^{6}$ Graduate Institute of Life Sciences, National Defense Medical Center, Taipei, Taiwan \\ 7 Brain Research Center, National Yang-Ming University, Taipei, Taiwan \\ ${ }^{8}$ Biophotonics and Molecular Imaging Research Center, National Yang-Ming University, Taipei, Taiwan \\ Correspondence to: Jin-Wu Tsai, email: tsaijw@ym.edu.tw
}

Bing-Wen Soong, email: bwsoong@ym.edu.tw

Keywords: PRRT2, neuronal migration, synaptic development, paroxysmal kinesigenic dyskinesia (PKD), Taiwan, Pathology Section Received: February 05, 2016

Accepted: April 26, 2016

Published: May 09, 2016

\section{ABSTRACT}

Mutations in the proline-rich transmembrane protein 2 (PRRT2) gene cause a wide spectrum of neurological diseases, ranging from paroxysmal kinesigenic dyskinesia (PKD) to mental retardation and epilepsy. Previously, seven PKD-related PRRT2 heterozygous mutations were identified in the Taiwanese population: P91QfsX, E199X, S202HfsX, R217PfsX, R217EfsX, R240X and R308C. This study aimed to investigate the disease-causing mechanisms of these PRRT2 mutations. We first documented that Prrt2 was localized at the pre- and post-synaptic membranes with a close spatial association with SNAP 25 by synaptic membrane fractionation and immunostaining of the rat neurons. Our results then revealed that the six truncating Prrt2 mutants were accumulated in the cytoplasm and thus failed to target to the cell membrane; the $\mathbf{R} 308 \mathrm{C}$ missense mutant had significantly reduced protein expression, suggesting loss-of function effects generated by these mutations. Using in utero electroporation of shRNA into cortical neurons, we further found that knocking down Prrt2 expression in vivo resulted in a delay in neuronal migration during embryonic development and a marked decrease in synaptic density after birth. These pathologic effects and novel disease-causing mechanisms may contribute to the severe clinical symptoms in PRRT2-related diseases.

\section{INTRODUCTION}

Mutations in the proline-rich transmembrane protein 2 gene (PRRT2, NM_145239.2) have been identified to be the causes of many neurological diseases. Heterozygous PRRT2 mutations cause paroxysmal kinesigenic dyskinesia (PKD), benign familial infantile epilepsy (BFIE), infantile convulsions and choreoathetosis
(ICCA), hemiplegic migraine (HM), episodic ataxia (EA), paroxysmal torticollis, or a combination of them [1-14]. Although rarely identified, patients with biallelic or homozygous PRRT2 mutations presented complex forms of PKD in combination with intellectual disability or cerebellar atrophy $[10,15,16]$. Previously, our group identified seven different PKD-related PRRT2 mutations in the Taiwanese population: p.P91Qfs*24 (P91QfsX), 
p.E199X (E199X), p.S202Hfs*16 (S202HfsX), p.R217Pfs*8 (R217PfsX), p.R217Efs*12 (R217EfsX), p.R240X (R240X) and p.R308C (R308C) [11, 17]. Six of these mutations result in premature truncation before the first predicted transmembrane domain. The only missense mutation, $\mathrm{R} 308 \mathrm{C}$, is located in the predicted cytoplasmic domain (Figure 1). There were a few in vitro studies suggesting that PRRT2 may interact with SNAP25 (the synaptosomal-associated protein, $25 \mathrm{kD}$ ), a critical molecule in neurotransmitter release [18, 19]. However, it is still not fully understood how mutated PRRT2 causes pathological effects on the nervous system in vivo.

Here, we generated a polyclonal antibody against Prrt2 which helped document that Prrt2 was localized at both the pre- and post-synaptic membranes with a close spatial association with SNAP25. We then revealed that abnormal intracellular trafficking and decreased expression of the aforementioned seven PRRT2 mutations could lead to its functional loss. Furthermore, by in utero electroporation of Prrt2 shRNA into mouse embryos, we showed that Prrt2 knockdown in cortical neurons caused a delay in neuronal migration and defects in synaptic development. These pathological effects may contribute to the severe clinical syndromes in patients with homozygous or biallelic PRRT2 mutations.

\section{RESULTS}

\section{Production of a highly specific Prrt2 polyclonal antibody}

To detect endogenous Prrt2, we first generated a polyclonal antibody against the extracellular domain of the Prrt2 protein and tested its specificity in rat and mouse brain lysates, as well as in COS-7 cells transfected with EGFP-Prrt2 construct (Figure 2A). The antibody showed high specificity for Prrt2, revealing a single major band $(\sim 65 \mathrm{kD})$ with few minor bands in each system using Western blotting. By using the antibody, endogenous Prrt2 was shown to localize at the cell membrane of mouse cortical neurons cultured for 3 or 16 days in vitro (DIV) (Figure 2B, left and middle panels). We also examined the distribution of EGFP-Prrt2 in transfected cultured HEK293T cells (Figure 2B, right panel). The fluorescence signals from EGFP and Prrt2 antibody predominantly colocalized at the cell membrane, consistent with previous observations using other protein tags $[1,20]$. To further test the specificity of the antibody, we test whether knocking down Prrt2 expression in cells by RNA interference (RNAi) will eliminate antibody reactivity (Figure 2C). Two short hairpin RNA (shRNA) plasmids targeting different Prrt 2 mRNA regions were used and showed 65\% (shPrrt2 \#1) and 98\% (shPrrt2 \#2) knockdown efficiencies 48 hours after transfection. We then transfected these shRNA constructs to cultured cortical neurons and found that Prrt2 signal was significantly lower than that in the surrounding non-transfected cells, indicating that the polyclonal antibody is highly specific.

\section{Close co-localization of Prrt 2 and SNAP25 on the pre- and post-synaptic membranes}

Synaptic membrane fractionation and postsynaptic density (PSD) preparation from adult rat brain lysates were carried out with several pre- and post-synaptic markers to confirm if Prrt2 is present on synapses (Figure 3A). Prrt2 was enriched in the synaptic membrane fraction (LP1), but was also detected in synaptic vesicle (LP2) and postsynaptic density (PSDI) fractions, indicating the presence of Prrt2 at both pre- and post-synaptic membranes. To further characterize the subcellular localization of Prrt2, we visualized the protein along dendritic spines in mature cultured hippocampal neurons

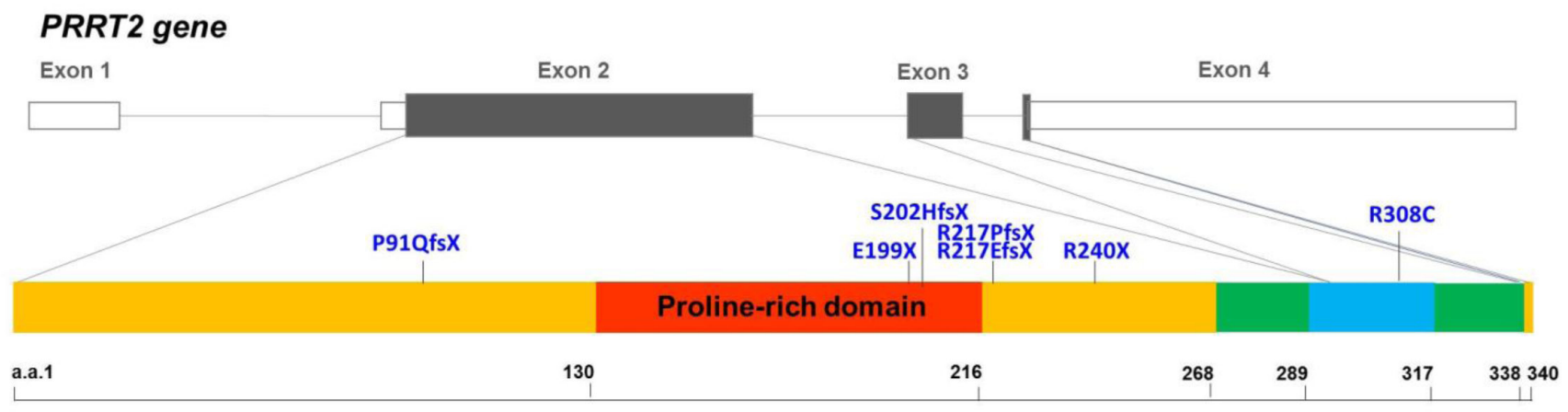

Figure 1: Genomic organization of the human PRRT2 gene and the distribution of the PRRT2 mutations identified in Taiwanese PKD patients. The genomic organization of human PRRT2 gene and the distribution of the seven PRRT2 mutations identified in Taiwanese PKD patients are illustrated in the upper panel. The predicted domains of the PRRT2 protein are shown in the lower panel. Six of the PRRT2 truncating mutations are localized in the predicted extracellular domain (amino acids 1-268), and five are clustered within or around the proline-rich domain (the red region). 


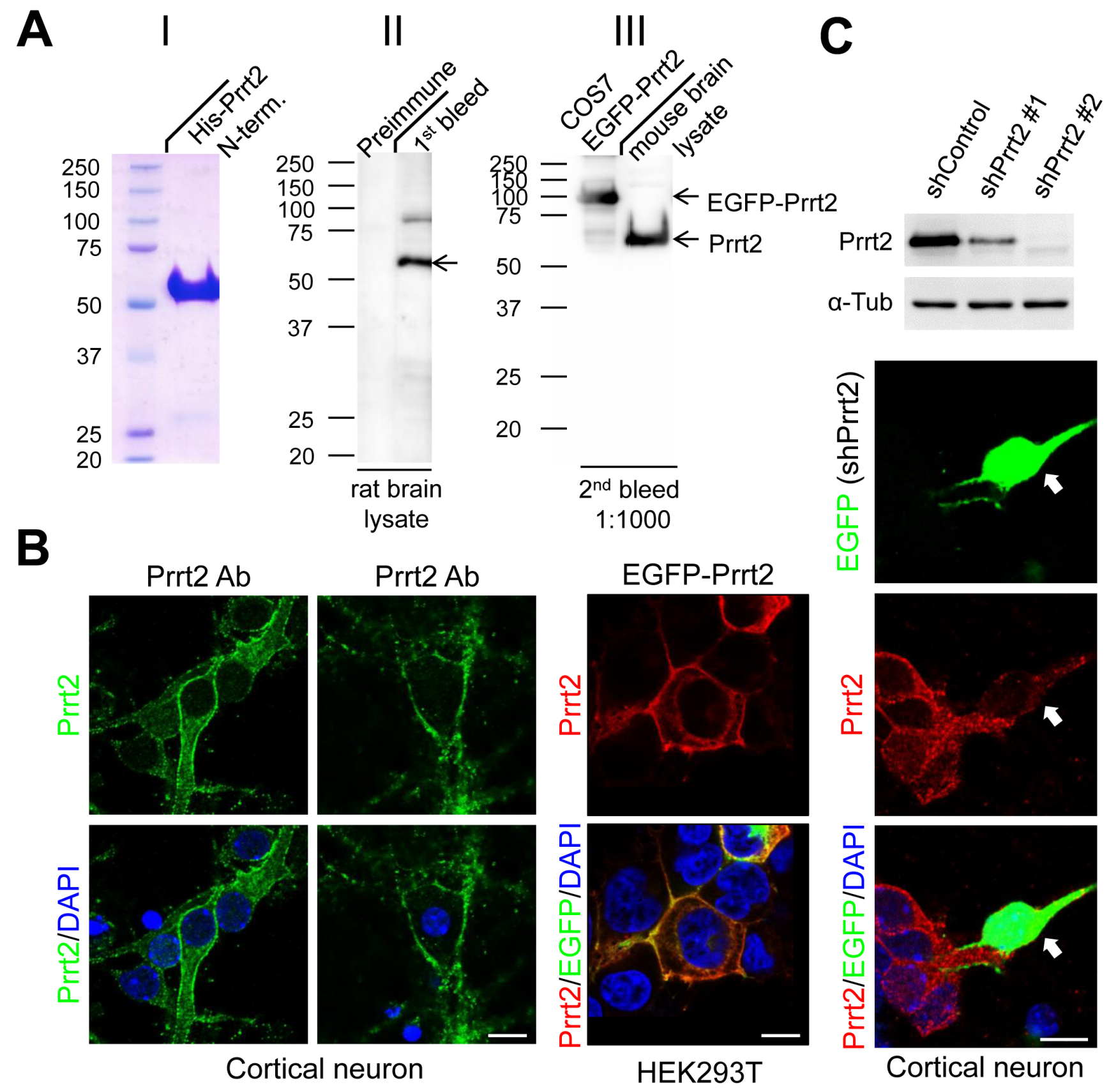

Figure 2: Prrt2 protein recognized by a specific polyclonal antibody. A. A polyclonal antibody against amino acids $1-268$ of the bacterially-expressed Prrt2 protein (I) was generated. Prrt2 protein from adult rat brain lysates was detected using the first bleed of Prrt2 anti-serum obtained from a rabbit (II, right lane). Preimmune serum drawn from the rabbit before antigen injection was the control (II, left lane). The antibody also recognized Prrt2 in mouse brain lysates and EGFP-tagged Prrt2 transfected into COS7 cells (III). B. Subcellular localization of Prrt2 protein by immunoflourescence staining with the polyclonal antibody. Endogenous Prrt2 protein (green) localized predominantly to cell membranes in cultured cortical neurons 3 and 16 days in vitro (DIV) (left panel). The distribution of EGFP-tagged Prrt2 also localized to the cell membrane in HEK293T cells transfected with EGFP-Prrt2, as revealed by the EGFP signal (green) and Prrt2 immuostaining (red). The distribution of Prrt2 based on EGFP signal and antibody staining was highly concordant and resembled the pattern of endogenous Prrt2 in cultured neurons. All cells were counterstained with DAPI staining (blue) to show the nuclei. Scale bar $=10 \mu \mathrm{m}$. C. Prrt2 protein level revealed by immunostaining with polyclonal antibody in cultured neurons transfected with Prrt2 shRNA. Upper panel: Prrt2 expression was knocked down by cotransfecting short hairpin RNA (shRNA) with Prrt2 into cultured cells. Two shRNA plasmids targeting different Prrt2 mRNA regions showed 65\% (shPrrt2 \#1) and 98\% (shPrrt2 \#2) knockdown efficiency 48 hours after transfection. Lower panel: Immunostaining in cultured neurons shows that endogenous Prrt2 signal (red) was significantly reduced in shPrrt2-transfected neurons (green, arrows) compared with the surrounding untransfected cells. All cells were counterstained with DAPI staining (blue) to show the nuclei. Scale bar $=10 \mu \mathrm{m}$. 
of adult rats. Prrt2 was visible at the tips of these spines where the majority of excitatory synapses are formed [21, 22], with a close spatial association with SNAP25 (Figure 3B).

\section{Failure of membrane targeting in truncated Prrt2 mutants}

The subcellular localization of the seven PKDrelated mutant Prrt2 was then explored in cultured cells

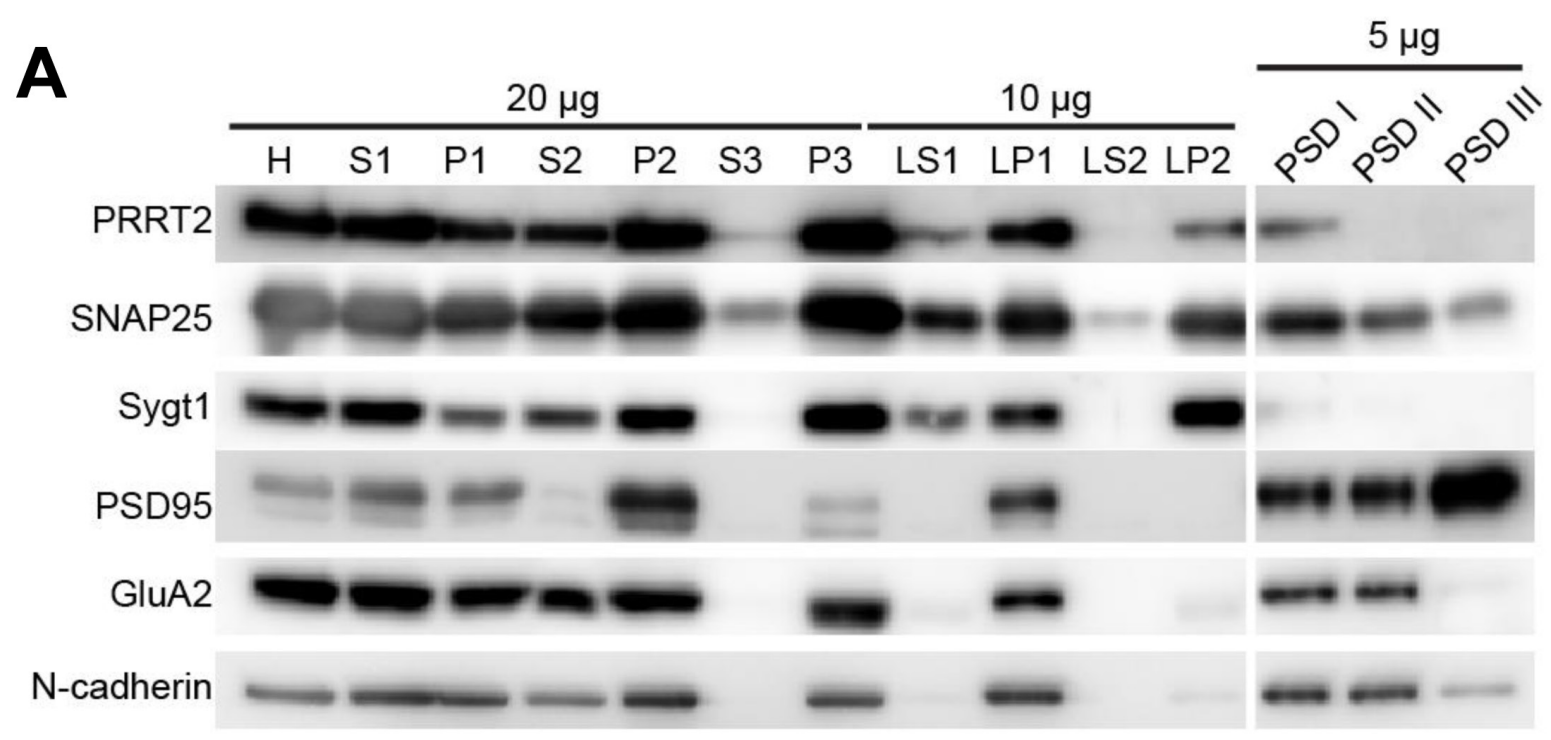

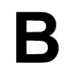

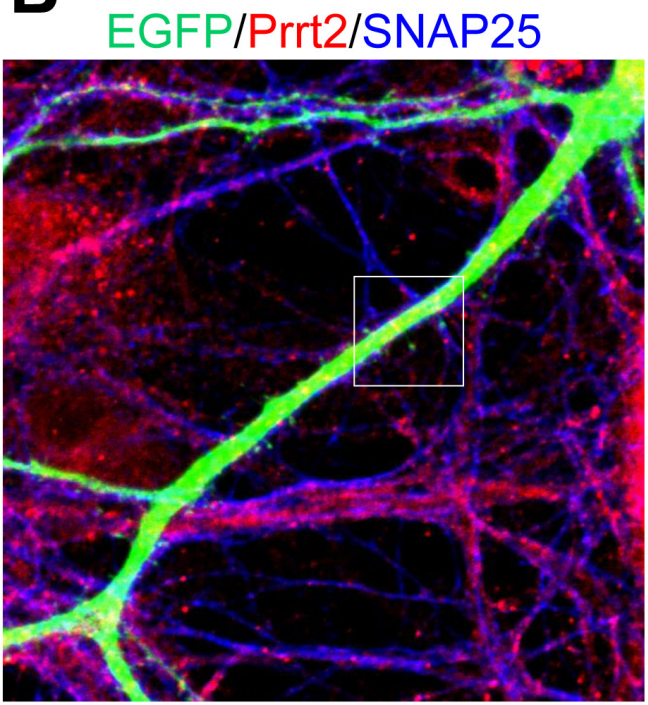

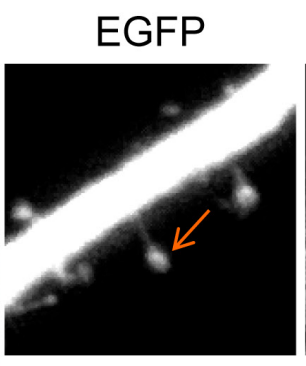

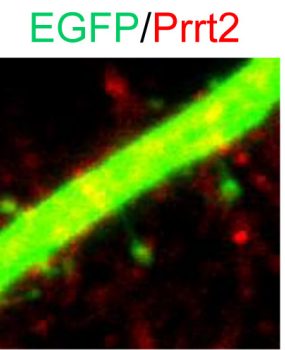

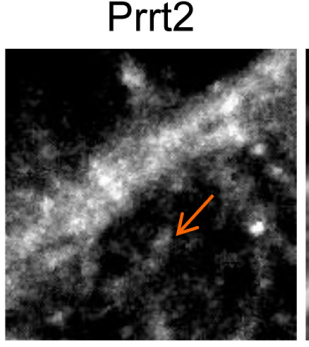
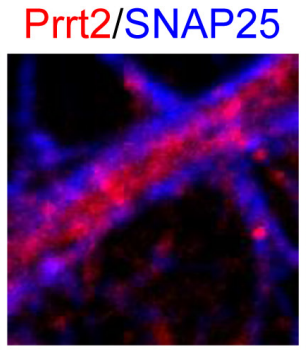

SNAP25

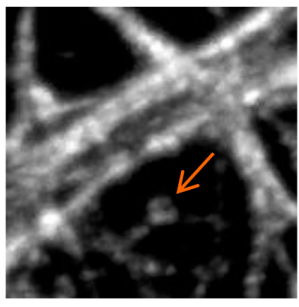

Merge

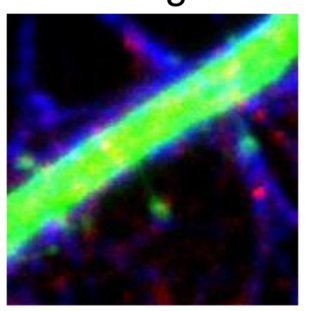

Figure 3: Localization of Prrt2 at the pre- and post-synaptic membranes. A. Membrane fractionation assay and post-synaptic density (PSD) preparation of the protein from an adult rat brain. Prrt2 and SNAP25 were present and co-fractionated in both pre- and postsynaptic compartments. PSD95 and GluA1 are PSD proteins that were enriched in PSD fractions. Synaptotagmin I (Sygt1)-a presynaptic vesicle protein was present in the LP2 fraction, but absent from all PSD fractions. PSD95, GluA2 and N-cadherin are post-synaptic membrane proteins that were absent from the LP2 fraction. $\mathrm{H}=$ homogenate, $\mathrm{S} 1=$ low-speed supernatant, $\mathrm{P} 1=$ nuclear pellet and debris, $\mathrm{S} 2=$ cytosol plus light membrane, P2 = crude synaptosomal fraction, S3 = cytosol, P3 = light membranes, LS1 = synaptosomal cytosolic fraction, LP1 = synaptosomal membrane fraction, LS2 = luminal fraction of synaptic vesicles, LP2 = synaptic vesicle membrane fraction. PSDI, II and III represent the detergent extractability of PSD resident proteins. PSD1 represents Single Triton X-100 extraction. PSDII and PSDIII were obtained in the second round of Triton extraction and Sarkosyl extraction of PSDI, respectively. B. Immunostaining of Prrt2 and SNAP25 in GFP-expressing cultured hippocampal neurons (DIV 17). A dendritic segment with multiple spines (specialized protrusive structures) is shown in the large image. The amplified images highlight the localization of Prrt2 (red) and SNAP25 (blue), which co-localized at the tip of a spine (orange arrow) where a synapse was forming. 
(Figure 4). HEK293T cells were transfected with EGFPPrrt 2 or its mutants and imaged with confocal microscopy. Wild type (WT) Prrt2 protein predominantly localized to the plasma membrane, consistent with the pattern observed for endogenous Prrt2 in cultured mouse neurons. In contrast, the six truncated Prrt 2 mutants were mostly retained in the cytoplasm, with some mutant proteins (P91QfsX, E199X and R240X) found in the nucleus, indicating abnormal intracellular trafficking of the mutant proteins. Of note, the R308C mutant was expressed on the plasma membrane in a pattern indistinguishable from WTPrrt2.

\section{EGFP-Prrt2 EGFP-Prrt2 + DAPI}
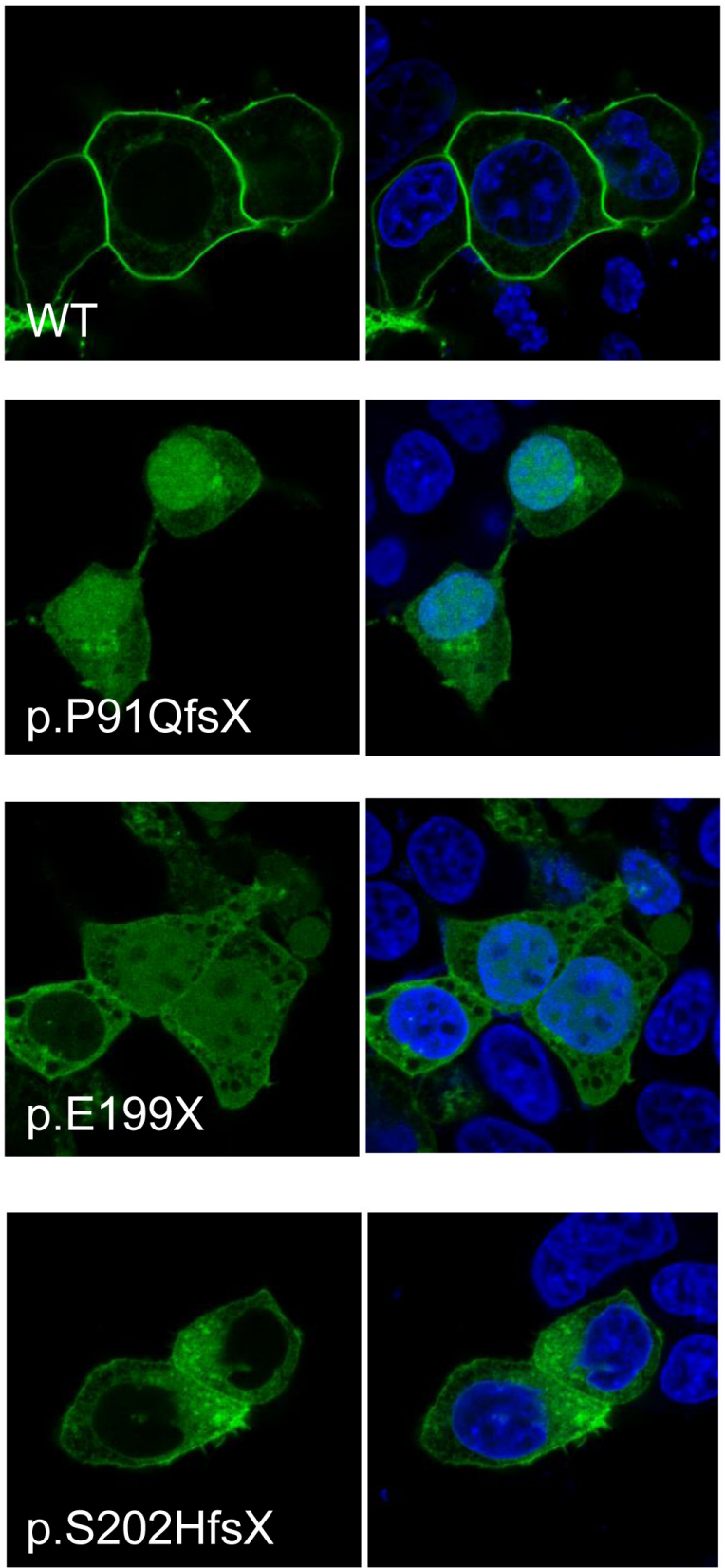

EGFP-Prrt2 EGFP-Prrt2 + DAPI
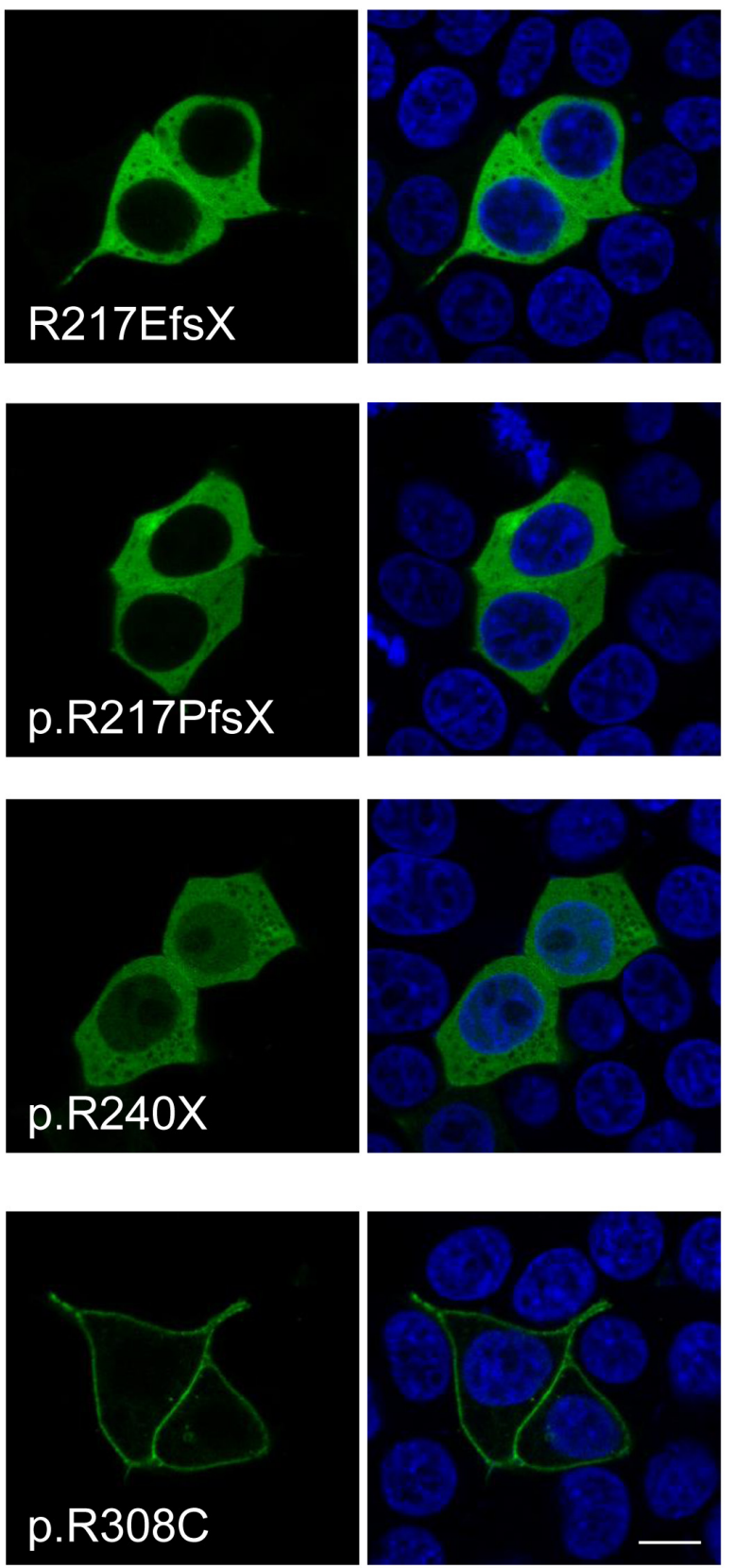

Figure 4: Subcellular localization of PKD-related PRRT2 mutations in cultured HEK293T cells. The six prematurely truncating Prrt2 mutants (P91QfsX, E199X, S202HfsX, R217EfsX, E217PfsX and R240X) were retained in the cytoplasm and failed to be properly transported to the membrane. Some faint signal from the p.P91QfsX, p.E199X and p.S202HfsX Prrt2 mutant proteins could be found in the nucleus. The missense R308C mutant protein had a membrane localization similar to the pattern for WT-Prrt2. Scale bar $=$ $10 \mu \mathrm{m}$. 


\section{Reduced Prrt2 protein level caused by the R308C mutation}

Although the R308C mutant was found to localize at the plasma membrane as WT, it is possible that insufficient level of mutant protein causes dysfunction of PRRT2. To address this possibility, EGFP- and HA-tagged WT-Prrt2 and the R308C mutant were transiently transfected to HEK293T cells and Prrt2 expression levels were examined by Western blotting. Significantly reduced protein levels were observed for the R308C mutant compared to WTPrrt2 (Figure 5), suggesting that this missense mutation may cause disease through reduced expression rather than abnormal trafficking.

\section{Delayed neuronal migration during development caused by Prrt2 knockdown}

To explore the loss-of-function effects of Prrt2 in the brain, we transfected Prrt2 shRNA constructs with cDNA expressing EGFP into mouse brains by in utero electroporation at embryonic (E) day 14.5 [23, 24]. Using this method, plasmids can be introduced into a subset of neural stem cells (i.e. radial glia) during cortical
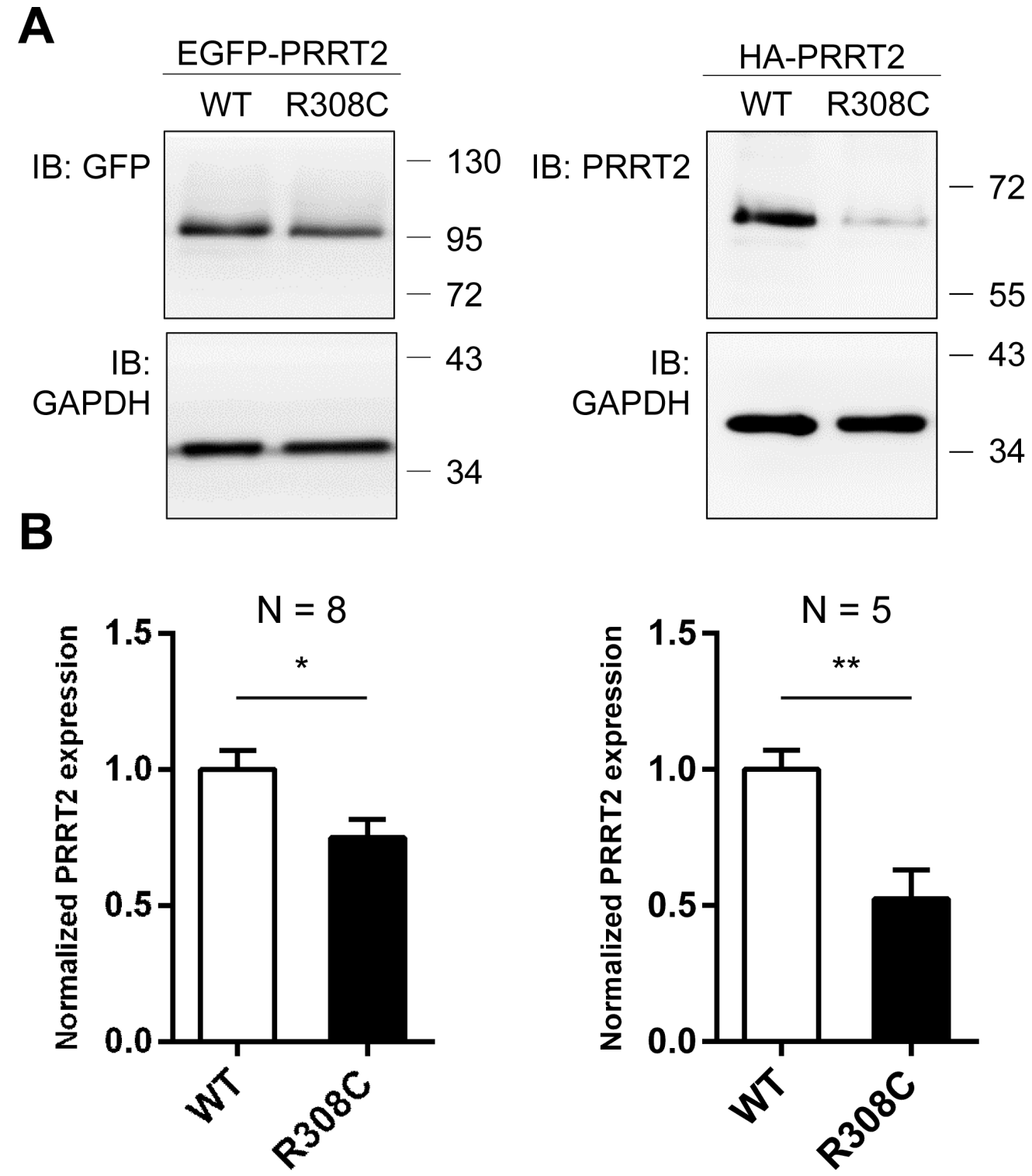

Figure 5: Reduced expression level of the R308C PRRT2 mutant. A. GFP- and HA-tagged WT-Prrt2 and the R308C mutant were transiently transfected into the HEK293T cell line. Prrt2 expression levels were determined using Western blotting and were normalized to that of WT-Prrt2. GAPDH was used as an internal control. B. A significant reduction of Prrt2 levels was observed for the R308C mutant (*: $p<0.05, * *: p<0.01)$. Error bars indicate SEM. Statistics were performed by a two-tailed, unpaired Student's t-test. 
development. The development of electroporated mouse brains was examined 3 and 5 days post electroporation, at E17.5 and postnatal (P) day 1, respectively. Normally, neurons generated from neural stem cells in the ventricular zone (VZ) migrate along radial fibers towards the cortical plate (CP) [25-27]. The neurons in the brains electroporated with Prrt2 shRNA exhibited a delay in neuronal migration from the $\mathrm{VZ}$ to the $\mathrm{CP}$, with many cells accumulated at the VZ, subventricular zone (SVZ) and intermediate zone (IZ) (Figure 6A, top panels). Quantification of the cells at day 3 (E17.5) revealed significantly reduced numbers of neurons in the $\mathrm{CP}$, as most of the cells remained in the VZ (Figure 6B). This migration delay was partially rescued by expressing Prrt2 in knockdown cells. Later, at day 5 (P1), most of the neurons in both the control and shRNA-electroporated brains reached the $\mathrm{CP}$ (Figure 6A, bottom panels), indicating that neuronal migration was delayed rather than completely blocked during cortical development.

\section{Decreased dendritic spine density caused by Prrt2 knockdown}

Since we documented the presence of Prrt2 at both the pre- and post-synaptic membranes, we hypothesized that loss of normal PRRT2 functions may cause changes in synaptic connections, which could be visualized by the density of dendritic spines [28]. Therefore, we investigated changes in dendritic spines in Prrt2 knockdown mice. Plasmids expressing Prrt2 shRNA and EGFP were electroporated into the developing cortex of mouse embryos in utero at E14.5. The mice were allowed to give birth and the brains of their pups were examined at P14, P21 and P30. Pyramidal neurons of layer $2 / 3$ (L2/3) were observed under a confocal or two-photon microscope. We counted the numbers of dendritic spines along apical dendrites (Figure 7A) [29]. At P14, neurons expressing Prrt2 shRNA showed a dendritic spine density similar to neurons in the control brains. However, there were significant decreases in spine density for neurons expressing shPrrt2 \#2 at P21 and P30 (Figure 7B). The reduction of dendritic spine density was not observed in the neurons expression shPrrt2 \#1 (data not shown). As shPrrt2 \#2 exhibited a much higher knockdown efficiency than shPrrt2 \#1 (Figure 2C), our results suggested that nearly complete knockdown of Prrt 2 may be required for the defect in dendritic spine during neural development and the loss-of-functional effect may be dose-dependent.

\section{DISCUSSION}

In this study, we identified important characteristics of the PRRT2 protein and its potential functions during neuronal and synaptic development. Using our highly specific antibody, we documented the localization of endogenous Prrt2 at both the pre- and post-synaptic membranes. PRRT2 mutations could lead to abnormal intracellular trafficking or reduced expression. More significantly, our in vivo functional assays using Prrt2 shRNAs showed that a nearly complete Prrt2 knockdown resulted in a delay in neuronal migration during early neural development and also defective spinogenesis. These results pointed to the essential functions of PRRT2 during neural development, which were not recognized before this study but may be critical in the pathomechanisms of PRRT2-related disorders.

Recent studies have revealed that PRRT2 is a component of the AMPAR ( $\alpha$-amino-3-hydroxy-5-methyl4 -isoxazolepropionic acid receptor) complex $[30,31]$ and may be involved in neurotransmitter release and glutamate signaling[20, 32]. Consistently, PRRT2 was found to colocalize with pre- and post-synaptic markers vGlut1 and PSD95 in our study. In addition, our study revealed that PRRT2 co-fractionated with SNAP25 and was localized close to it at synapses, supporting the interactions between these two proteins.

We observed a reduced protein level in the HEK293T cells expressing the R308C mutant. Reduced expression has been reported in cells expressing other PRRT2 mutations $[19,33,34]$ and could be a common functional loss of PKD-related mutations. It remains unclear whether the decreased protein level in cells was caused by decreased production, protein instability, or both. Either way, this result suggested that haploinsufficiency resulting from some forms of PRRT2 mutations may be the pathomechanism of PKD-related disorders. Another loss-of-function effect underlying PKD and related diseases was seen in the truncated forms of Prrt2, which were retained in the cytoplasm with some entering the nucleus.

Using in utero electroporation, we found that a further decrease in PRRT2 expression in cortical neurons resulted in delayed neuronal migration in the early stage of development. Furthermore, nearly complete Prrt2 knockdown caused a reduction in dendritic spine density. Consistent with this result, a recent in vitro study also showed a decrease in synapses in cultured hippocampal neurons when Prrt2 was knocked down [32]. Delayed neuronal migration and abnormal spine density are frequently associated with various cognitive, learning and memory deficits [28, 34-36]. Our results indicated that these defects may contribute to the severe encephalopathy caused by the rare homozygous or biallelic PRRT2 mutations [10, 15, 16, 37].

Together, we proposed the potential mechanism that may underlie various PRRT2-related diseases (Figure 8). PRRT2 participates in normal synaptic functions through interactions with SNAP25 [18, 19, 32] and probably other molecules of the AMPAR complex [30, 31]. PRRT2 mutations cause reduced expression and/or intracellular mislocalization of PRRT2 proteins, leading 

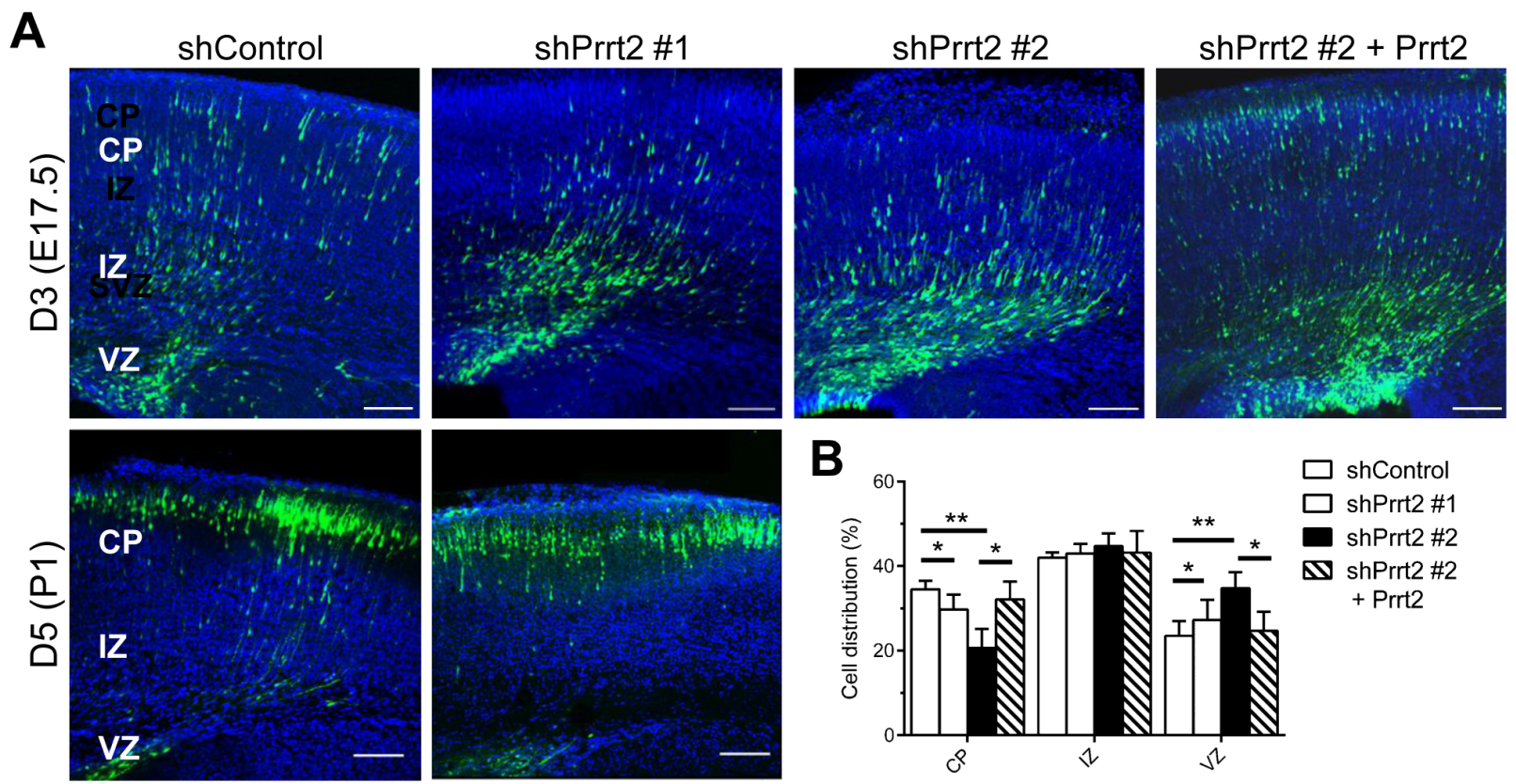

Figure 6: Delay in neuronal migration by Prrt2 knockdown during development. A. Coronal sections of the mouse brains 3 and 5 days after in utero electroporation of shControl or shPrrt2 constructs at E14.5. Three days after electroporation, cells expressing EGFP (green) and shControl had started migration, with many cells having already reached the CP. More cells were restricted to the VZ and IZ in the brains electroporated with each of the shPrrt2. This migration delay was rescued by co-transfection of shPrrt2 and Prrt2 constructs (upper panel). When brain sections were examined 5 days post electroporation, most cells in both shControl and shPrrt2 groups had reached the CP (lower panel). All sections were stained with DAPI (blue) to show the cell nuclei. Scale bars $=100 \mu \mathrm{m}$. B. Statistical analysis showed significant differences in cell distribution in the CP, IZ and VZ at E17.5for the four conditions. Error bars represent SEM; $*: p<0.05, * *: p<0.01$.

A

$\mathrm{P} 21$

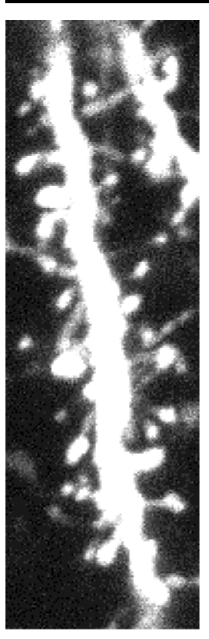

shControl

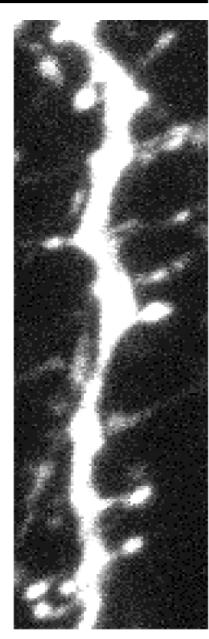

shPrrt2 \#2

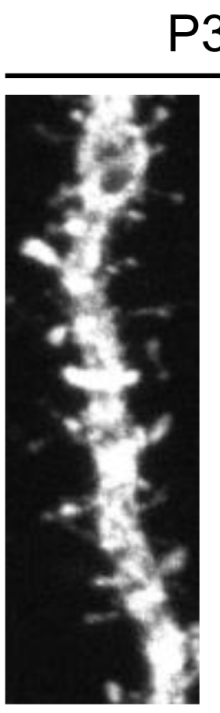

shControl

P30

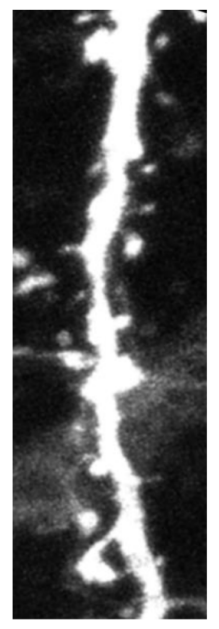

shPrrt2 \#2
B

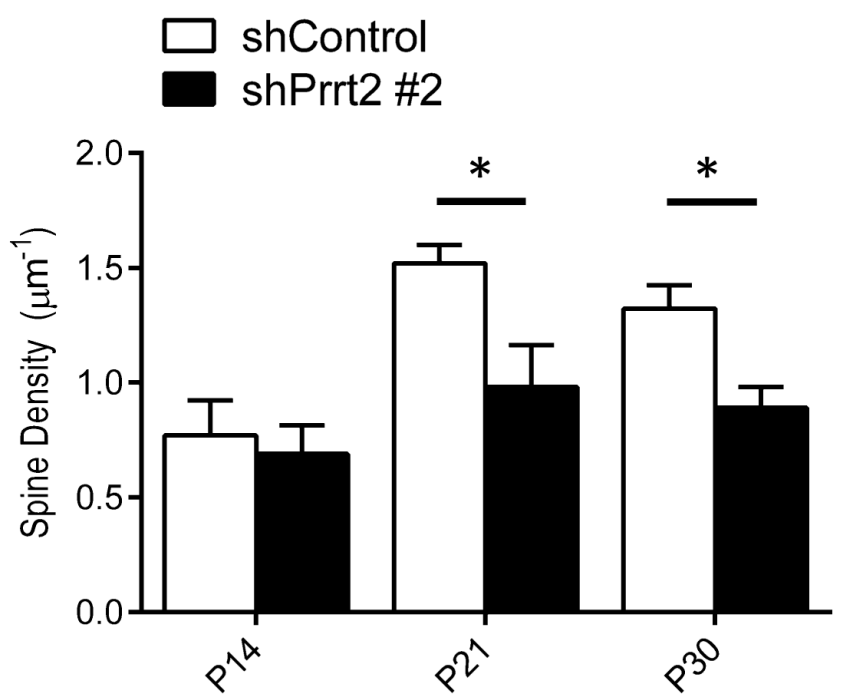

Figure 7: Decreased dendritic spine density after Prrt2 knockdown. A. Dendritic spines were imaged at P21 and P30 in brains electroporated in utero with control or Prrt2 shRNA along with EGFP constructs at E14.5. Lower spine densities for Prrt2 shRNAexpressing pyramidal neurons were observed on representative dendrites at both P21 and P30. B. Statistical analysis of spine density of dendrites at P14, P21 and P30. There was no significant difference between control and Prrt2 shRNA-expressing groups at P14. Significant decreases in spine density for Prrt2 shRNA-expressing cells were observed at P21 and P30. *: $p<0.05$. 


\section{Normal}

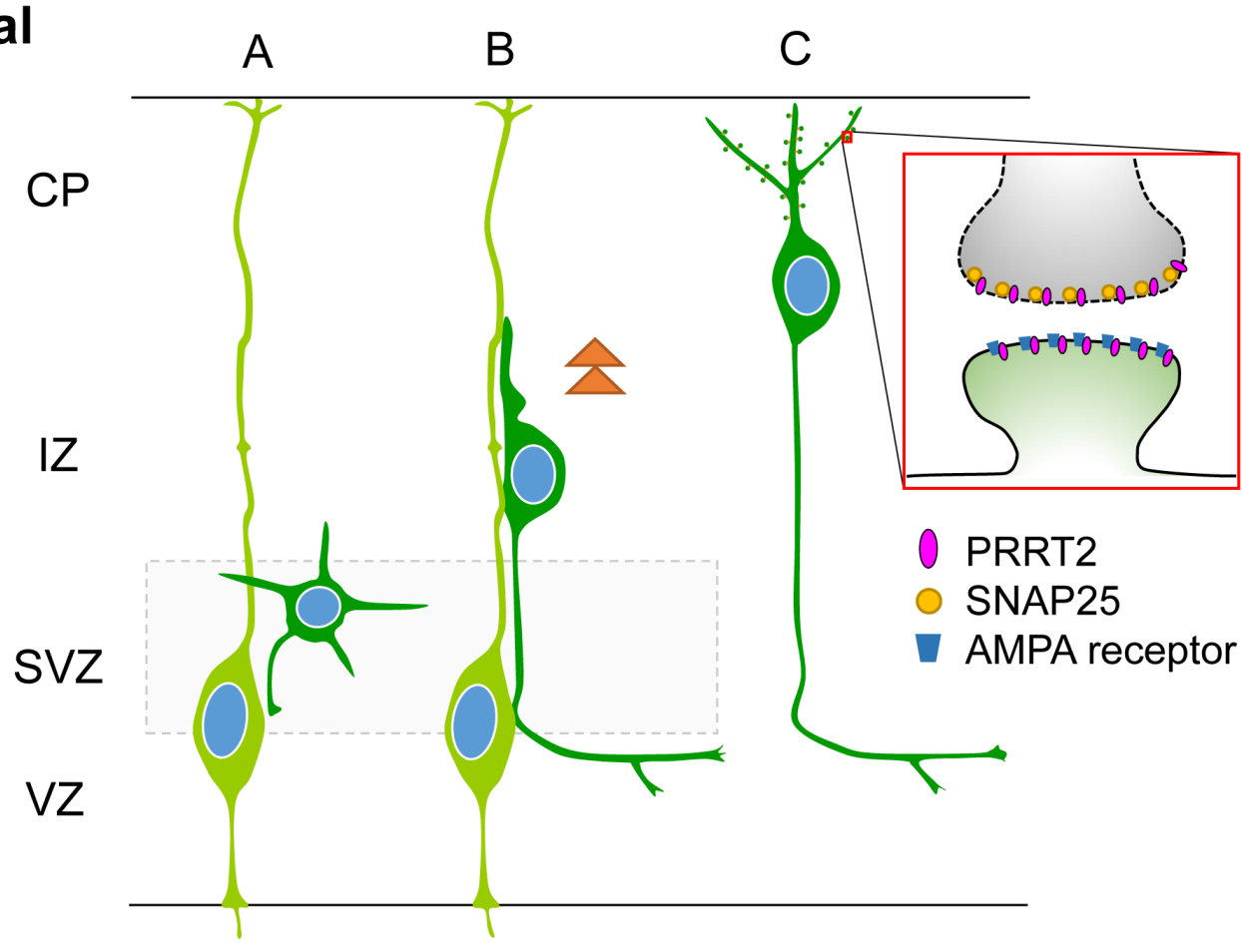

\section{PRRT2 mutants}

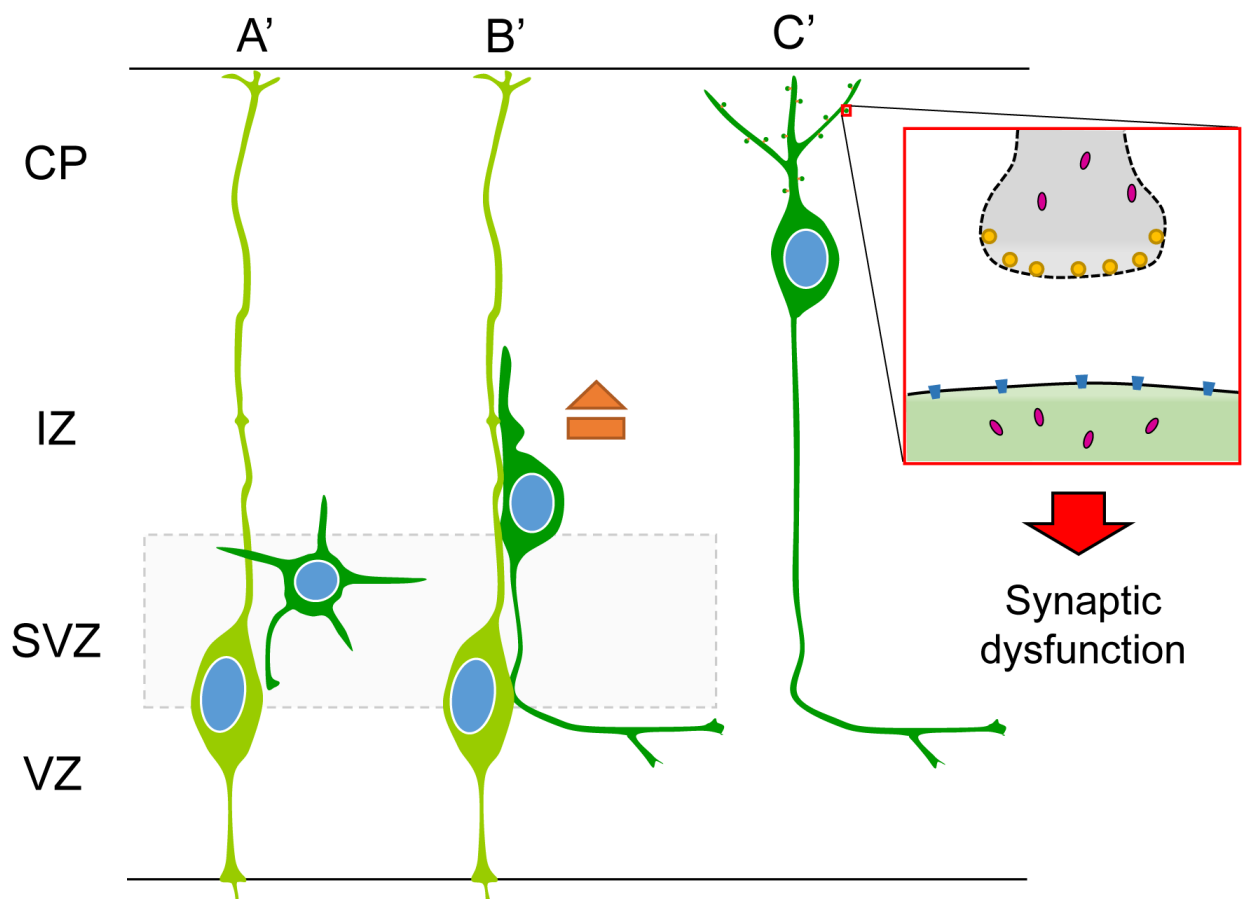

Figure 8: Schematic diagram showing effects of PRRT2 mutation on neuronal migration and dendritic spines in cortical neurons. A. Radial glia cells (light green) produce postmitotic neurons (dark green). B. Postmitotic neurons then migrate along the radial fiber to the CP. C. After reaching the cortical plate, the cells extend dendrites and form synaptic connections with other neurons through dendritic spines. Box shows a magnification of the pre- and post-synaptic regions. PRRT2 (pink) is localized at both the pre- and post-synaptic membranes through interactions with SNAP25 $5^{18,25,44}$ and the AMPA receptor ${ }^{25,33,34}$. (A', B') In neurons carrying PKDassociated PRRT2 mutations, reduced expression, mislocalization and other potential defects delay the neuronal migration. (C') PRRT2 mutation also decreases the dendritic spine density and causes synaptic dysfunction, possibly through SNAP25 and/or AMPA receptor pathways. 
to their functional loss. PRRT2 loss-of-function can also lead to a delay in neuronal migration and synaptic loss. The diverse pathological impacts of PRRT2 mutations in turn contribute to the wide phenotypic spectrum, from PKD by heterozygous mutations to severe congenital encephalopathy by homozygous or biallelic mutations.

\section{MATERIALS AND METHODS}

\section{Antibody production}

The Prrt2 antibody was generated using antigen prepared by Nickel resin purification from a bacteriallyexpressed peptide comprising the extracellular domain (amino acids 1-268) of Prrt2 protein with a 6X His tag. Prrt 2 proteins from adult rat brain lysates were detected using the first bleed of Prrt2 anti-serum obtained from a rabbit, whereas the preimmune serum was drawn from the rabbit before antigen injection as the control.

\section{Cell culture and transient transfection}

Cultured hippocampal and cortical neurons were isolated from P0 Sprague-Dawley rats and E18 ICR mice, respectively, according to our previous report [38]. COS-7 (monkey kidney epithelial cells) and HEK293T (human embryonic kidney cells) cell lines were cultured in Dulbecco's Modified Eagle Medium (DMEM, Life Technologies, USA) supplemented with $10 \%$ fetal bovine serum. Transfection was carried out using the Lipofectamine 2000 and 3000 lipofection reagents (Life Technologies, USA) and cells were harvested 24-48 hours after transfection.

\section{Plasmids and antibodies}

The mouse Prrt2 cDNA construct was purchased from Origene (MG214094; GenBank Accession No.: NM_01102563). The enhanced green fluorescent proteintagged Prrt2 (EGFP-Prrt2) constructs of the wild-type Prrt2 and the 7 PKD-associated mutations were generated and cloned into the pEGFP-C1 vector (Clontech, Palo Alto, CA). The human influenza hemagglutinin-tagged Prrt2 (HA-Prrt2) constructs were cloned into the pcDNA3.1 (-) vector (Clontech, Palo Alto, CA). All mutant constructs were fully sequenced to verify the mutated nucleotides.

\section{Western blot analysis}

EGFP-Prrt2 and HA-Prrt2 constructs with either wild-type or the point mutation, R308C, were transiently transfected into an HEK293 cell line. Total proteins were harvested at 24 hours after transfection. The protein concentrations were determined using a BCA assay (Thermo Fisher Scientific, USA). The results were quantified using ImageJ software (National Institutes of Health, NIH, USA).

\section{Knock-down constructs}

For RNAi, short hairpin RNA (shRNA) constructs were based on the pLKO.1-puro vector (RNAi core of Academia Sinica, Taiwan) and pGPU6/GFP/Neo vector (Tools, Taiwan). The Prrt2 target sequences (shPrrt2 \#1: 5'-CATCAACTTAGGCGTGTATAA-3' and shPrrt2 \#2: 5'-GGCCACAGACCTCAGTTTAAA-3') were chosen from a portion of the Prrt2 coding region, where the sequence was conserved between human and mouse. The empty vector or a scramble (5'-TTCTCCGAACGTGTCACGT-3') sequence (shControl) was used as the control.

\section{Immunofluorescence}

Cultured mice neurons were fixed in $4 \%$ paraformaldehyde $/ 4 \%$ sucrose/ phosphate-buffered saline (PBS) solution for 20 minutes at $4^{\circ} \mathrm{C}$, followed by PBS rinsing and $0.1 \%$ Triton $\mathrm{X}-100$ permeabilization at $4^{\circ} \mathrm{C}$ for 15 minutes. Neurons were then incubated at room temperature (RT) for 20 minutes in blocking solution ( $2 \%$ $\mathrm{BSA} / 4 \%$ normal goat serum/PBS). The primary antibodies were diluted in blocking solution and added to neurons for incubation at $4{ }^{\circ} \mathrm{C}$ overnight in a humidified chamber, followed by secondary antibody incubation in the dark at RT for 1 hour. Coverslips were mounted in Prolonged Gold Antifade reagent (Life Technologies, USA) and stored at $4{ }^{\circ} \mathrm{C}$ until imaging.

\section{Image acquisition}

HEK293T cells expressing wild-type or mutated Prrt 2 proteins were imaged by a confocal microscope (Zeiss LSM700, Germany) with a 100X Plan-Apochromat $\mathrm{NA}=1.4$ oil objective. Final composite images were processed by the ImageJ software (NIH, USA).

\section{Membrane fractionation and post-synaptic density (PSD) purification}

The adult rat brain was dissected out for subcellular fractionation as previously described [39]. PSD preparation was performed as described previously [40]. 


\section{In utero electroporation}

Plasmids were transfected using intraventricular injection of embryonic ICR mice in utero, followed by electroporation as previously described [26]. Embryos were then perfused transcardially using cold PBS (pH 7.4) followed by $4 \%$ paraformaldehyde in PBS. Brains were collected and further postfixed in 4\% paraformaldehyde overnight at $4{ }^{\circ} \mathrm{C}$. For brain slice preparation, brains were embedded in 4\% low-melting-point agarose (Amresco, USA) dissolved in PBS and sectioned using a Vibratome (Leica, Germany) at a thickness of $100 \mu \mathrm{m}$.

\section{Dendritic spine analysis}

Mouse brains electroporated with EGFP were fixed, sectioned and imaged with a confocal or two-photon microscope equipped with a 40X objective lens (Zeiss, Germany) as previously described [29]. Apical dendrites extending from the layer (L) 2/3 pyramidal neurons expressing GFP were randomly selected and analyzed semi-automatically with a customized Matlab program. All image analysis and quantification was conducted in a double-blind manner.

Animals were maintained according to protocols approved by the Institutional Animal Care and Use Committee (IACUC) at National Yang-Ming University. All animal studies were conducted in accordance with the United States Public Health Service's Policy on Humane Care and Use of Laboratory Animals.

\section{Abbreviations}

BFIE: benign familial infantile epilepsy; CP: cortical plate; DIV: days in vitro; E: embryonic; EA: episodic ataxia; EGFP: enhanced green fluorescent protein; HA: human influenza hemagglutinin; HM: hemiplegic migraine; ICCA: infantile convulsions and choreoathetosis; IZ: intermediate zone; PKD: paroxysmal kinesigenic dyskinesia; P: postnatal; PRRT2: the prolinerich transmembrane protein 2 gene; PRRT2: the prolinerich transmembrane protein; PSD: postsynaptic density; RNAi: RNA interference; shRNA: short hairpin RNA; SNAP25: the synaptosomal-associated protein, 25kD; SVZ: subventricular zone; VZ: ventricular zone

\section{ACKNOWLEDGEMENTS}

YTL is grateful for the funding from the National Health Research Institutes (NHRI-EX105-10507EC) and Taipei Veterans General Hospital (V104B-015 and V105C-166). She also thank for the Clinical Research Core Laboratory of Taipei Veterans General Hospital for providing experimental space and facilities. CYT is grateful to the Development Center for Biotechnology and Academia Sinica for support. BWS is grateful for the funding support from the Ministry of Science and Technology (MOST 103-2314-B-010-049-MY3, MOST 104-2745-B-010-004, MOST 104-2745-B-010-002), Brain Research Center of National Yang-Ming University (104AC-B19) and Taipei Veterans General Hospital (V103C-109, V104E9-006). JWT is grateful to the Ministry of Science and Technology (101-2320-B-010077-MY2，103-2911-I-010-504，103-2628-B-010-002MY3, 104-2633-H-010-001, and 105-2633-B-009-003), Taiwan National Health Research Institutes (NHRIEX103-10314NC), and Academia Sinica, Taiwan (AS104-TP-B09).

\section{Author contributions}

Dr. Yo-Tsen Liu drafted the manuscript and Figureures, acquired and analyzed the data.

Ms. Fang-Shin Nian drafted the manuscript and Figureures, acquired and analyzed the data.

Ms. Wan-Ju Chou acquired and analyzed the data and revised the manuscript.

Dr. Chin-Yin Tai acquired and analyzed the data and revised the manuscript.

Dr. Shang-Yeong Kwan acquired and analyzed the data and revised the manuscript.

Dr. Chien Chen acquired and analyzed the data and revised the manuscript.

Ms. Pei-Wen Kuo acquired and analyzed the data and revised the manuscript.

Mr. Po-Hsi Lin acquired and analyzed the data and revised the manuscript.

Ms. Chin-Yi Chen acquired and analyzed the data and revised the manuscript.

Mr. Chia-Wei Huang acquired and analyzed the data and revised the manuscript.

Dr. Yi-Chung Lee acquired and analyzed the data and revised the manuscript.

Prof. Bing-Wen Soong contributed to design and conceptualized the study, interpreted the data, revised the manuscript and supervised the study.

Dr. Jin-Wu Tsai contributed to the design and conceptualization of the study, conducted the statistical analysis, interpreted the data, revised the manuscript and supervised the study.

\section{CONFLICTS OF INTEREST}

All authors declare no conflicts of interest. 


\section{REFERENCES}

1. Chen WJ, Lin Y, Xiong ZQ, Wei W, Ni W, Tan GH, Guo SL, He J, Chen YF, Zhang QJ, Li HF, Murong SX, $\mathrm{Xu}$ J, Wang $\mathrm{N}$ and $\mathrm{Wu} \mathrm{ZY}$. Exome sequencing identifies truncating mutations in PRRT2 that cause paroxysmal kinesigenic dyskinesia. Nature genetics. 2011; 43:12521255 .

2. Cloarec R, Bruneau N, Rudolf G, Massacrier A, Salmi M, Bataillard M, Boulay C, Caraballo R, Fejerman N, Genton P, Hirsch E, Hunter A, Lesca G, Motte J, Roubertie A, Sanlaville D, et al. PRRT2 links infantile convulsions and paroxysmal dyskinesia with migraine. Neurology. 2012; 79:2097-2103.

3. Dale RC, Gardiner A, Antony J and Houlden H. Familial PRRT2 mutation with heterogeneous paroxysmal disorders including paroxysmal torticollis and hemiplegic migraine. Dev Med Child Neurol. 2012; 54:958-960.

4. Gardiner AR, Bhatia KP, Stamelou M, Dale RC, Kurian MA, Schneider SA, Wali GM, Counihan T, Schapira AH, Spacey SD, Valente EM, Silveira-Moriyama L, Teive HA, Raskin S, Sander JW, Lees A, et al. PRRT2 gene mutations: from paroxysmal dyskinesia to episodic ataxia and hemiplegic migraine. Neurology. 2012; 79:2115-2121.

5. Marini C, Conti V, Mei D, Battaglia D, Lettori D, Losito E, Bruccini G, Tortorella G and Guerrini R. PRRT2 mutations in familial infantile seizures, paroxysmal dyskinesia, and hemiplegic migraine. Neurology. 2012; 79(21):2109-2114.

6. Scheffer IE, Grinton BE, Heron SE, Kivity S, Afawi Z, Iona X, Goldberg-Stern H, Kinali M, Andrews I, Guerrini R, Marini C, Sadleir LG, Berkovic SF and Dibbens LM. PRRT2 phenotypic spectrum includes sporadic and feverrelated infantile seizures. Neurology. 2012; 79:2104-2108.

7. Schubert J, Paravidino R, Becker F, Berger A, Bebek N, Bianchi A, Brockmann K, Capovilla G, Dalla Bernardina B, Fukuyama Y, Hoffmann GF, Jurkat-Rott K, Anttonen AK, Kurlemann G, Lehesjoki AE, Lehmann-Horn F, et al. PRRT2 mutations are the major cause of benign familial infantile seizures. Hum Mutat. 2012; 33:1439-1443.

8. Brockmann K. Episodic movement disorders: from phenotype to genotype and back. Curr Neurol Neurosci Rep. 2013; 13:379.

9. Heron SE and Dibbens LM. Role of PRRT2 in common paroxysmal neurological disorders: a gene with remarkable pleiotropy. J Med Genet. 2013; 50:133-139.

10. Labate A, Tarantino P, Palamara G, Gagliardi M, Cavalcanti F, Ferlazzo E, Sturniolo M, Incorpora G, Annesi G, Aguglia $\mathrm{U}$ and Gambardella A. Mutations in PRRT2 result in familial infantile seizures with heterogeneous phenotypes including febrile convulsions and probable SUDEP. Epilepsy Res. 2013; 104:280-284.

11. Chou IC, Lin SS, Lin WD, Wang CH, Chang YT, Tsai FJ and Tsai CH. Successful control with carbamazepine of family with paroxysmal kinesigenic dyskinesia of PRRT2 mutation. Biomedicine (Taipei). 2014; 4:15.

12. Mao CY, Shi CH, Song B, Wu J, Ji Y, Qin J, Li YS, Wang JJ, Shang DD, Sun SL and Xu YM. Genotype-phenotype correlation in a cohort of paroxysmal kinesigenic dyskinesia cases. J Neurol Sci. 2014; 340:91-93.

13. Tan LC, Methawasin K, Teng EW, Ng AR, Seah SH, Au WL, Liu JJ, Foo JN, Zhao Y and Tan EK. Clinico-genetic comparisons of paroxysmal kinesigenic dyskinesia patients with and without PRRT2 mutations. European journal of neurology. 2014; 21:674-678.

14. Huang XJ, Wang T, Wang JL, Liu XL, Che XQ, Li J, Mao X, Zhang M, Bi GH, Wu L, Zhang Y, Wang JY, Shen JY, Tang BS, Cao L and Chen SD. Paroxysmal kinesigenic dyskinesia: Clinical and genetic analyses of 110 patients. Neurology. 2015; 85:1546-1553.

15. Delcourt M, Riant F, Mancini J, Milh M, Navarro V, Roze E, Humbertclaude V, Korff C, Des Portes V, Szepetowski P, Doummar D, Echenne B, Quintin S, Leboucq N, Singh Amrathlal R, Rochette J, et al. Severe phenotypic spectrum of biallelic mutations in PRRT2 gene. Journal of neurology, neurosurgery, and psychiatry. 2015; 86:782-785.

16. Meneret A, Gaudebout C, Riant F, Vidailhet M, Depienne $\mathrm{C}$ and Roze E. PRRT2 mutations and paroxysmal disorders. European journal of neurology. 2013; 20:872-878.

17. Lee YC, Lee MJ, Yu HY, Chen $\mathrm{C}$, Hsu CH, Lin KP, Liao KK, Chang MH, Liao YC and Soong BW. PRRT2 mutations in paroxysmal kinesigenic dyskinesia with infantile convulsions in a Taiwanese cohort. PLoS One. 2012; 7:e38543.

18. Stelzl U, Worm U, Lalowski M, Haenig C, Brembeck FH, Goehler H, Stroedicke M, Zenkner M, Schoenherr A, Koeppen S, Timm J, Mintzlaff S, Abraham C, Bock N, Kietzmann S, Goedde A, et al. A human protein-protein interaction network: a resource for annotating the proteome. Cell. 2005; 122:957-968.

19. Lee HY, Huang Y, Bruneau N, Roll P, Roberson ED, Hermann M, Quinn E, Maas J, Edwards R, Ashizawa T, Baykan B, Bhatia K, Bressman S, Bruno MK, Brunt ER, Caraballo R, et al. Mutations in the gene PRRT2 cause paroxysmal kinesigenic dyskinesia with infantile convulsions. Cell Rep. 2012; 1:2-12.

20. Li M, Niu F, Zhu X, Wu X, Shen N, Peng X and Liu Y. PRRT2 Mutant Leads to Dysfunction of Glutamate Signaling. Int J Mol Sci. 2015; 16:9134-9151.

21. Christensen BN. Distribution of electrotonic synapses on identified lamprey neurons: a comparison of a model prediction with an electron microscopic analysis. J Neurophysiol. 1983; 49:705-716.

22. Christensen BN and Teubl WP. Localization of synaptic input on dendrites of a lamprey spinal cord neurone from physiological measurements of membrane properties. J Physiol. 1979; 297:319-333.

23. Saito $T$ and Nakatsuji N. Efficient gene transfer into the embryonic mouse brain using in vivo electroporation. 
Developmental biology. 2001; 240:237-246.

24. Tabata $\mathrm{H}$ and Nakajima K. Efficient in utero gene transfer system to the developing mouse brain using electroporation: visualization of neuronal migration in the developing cortex. Neuroscience. 2001; 103:865-872.

25. Noctor SC, Flint AC, Weissman TA, Dammerman RS and Kriegstein AR. Neurons derived from radial glial cells establish radial units in neocortex. Nature. 2001; 409:714720.

26. Tsai JW, Bremner KH and Vallee RB. Dual subcellular roles for LIS1 and dynein in radial neuronal migration in live brain tissue. Nature neuroscience. 2007; 10:970-979.

27. Tsai JW, Chen Y, Kriegstein AR and Vallee RB. LIS1 RNA interference blocks neural stem cell division, morphogenesis, and motility at multiple stages. The Journal of cell biology. 2005; 170:935-945.

28. Kasai H, Matsuzaki M, Noguchi J, Yasumatsu N and Nakahara H. Structure-stability-function relationships of dendritic spines. Trends Neurosci. 2003; 26:360-368.

29. Ma L, Qiao Q, Tsai JW, Yang G, Li W and Gan WB. Experience-dependent plasticity of dendritic spines of layer $2 / 3$ pyramidal neurons in the mouse cortex. Dev Neurobiol. 2016; 76:277-286.

30. Schwenk J, Harmel N, Brechet A, Zolles G, Berkefeld H, Muller CS, Bildl W, Baehrens D, Huber B, Kulik A, Klocker N, Schulte U and Fakler B. High-resolution proteomics unravel architecture and molecular diversity of native AMPA receptor complexes. Neuron. 2012; 74:621633.

31. Shanks NF, Savas JN, Maruo T, Cais O, Hirao A, Oe S, Ghosh A, Noda Y, Greger IH, Yates JR, 3rd and Nakagawa T. Differences in AMPA and kainate receptor interactomes facilitate identification of AMPA receptor auxiliary subunit GSG1L. Cell Rep. 2012; 1:590-598.

32. Valente P, Castroflorio E, Rossi P, Fadda M, Sterlini B, Cervigni RI, Prestigio C, Giovedi S, Onofri F, Mura E, Guarnieri FC, Marte A, Orlando M, Zara F, Fassio A, Valtorta F, et al. PRRT2 Is a Key Component of the CaDependent Neurotransmitter Release Machinery. Cell Rep. 2016; 15:117-131.
33. Gardiner AR, Jaffer F, Dale RC, Labrum R, Erro R, Meyer E, Xiromerisiou G, Stamelou M, Walker M, Kullmann D, Warner T, Jarman P, Hanna M, Kurian MA, Bhatia KP and Houlden $\mathrm{H}$. The clinical and genetic heterogeneity of paroxysmal dyskinesias. Brain. 2015; 138:3567-3580.

34. Ji Z, Su Q, Hu L, Yang Q, Liu C, Xiong J and Xiong F. Novel loss-of-function PRRT2 mutation causes paroxysmal kinesigenic dyskinesia in a Han Chinese family. BMC Neurol. 2014; 14:146.

35. Penzes P, Cahill ME, Jones KA, VanLeeuwen JE and Woolfrey KM. Dendritic spine pathology in neuropsychiatric disorders. Nature neuroscience. 2011; 14:285-293.

36. Jiang M, Ash RT, Baker SA, Suter B, Ferguson A, Park J, Rudy J, Torsky SP, Chao HT, Zoghbi HY and Smirnakis SM. Dendritic arborization and spine dynamics are abnormal in the mouse model of MECP2 duplication syndrome. The Journal of neuroscience. 2013; 33:1951819533.

37. Najmabadi H, Hu H, Garshasbi M, Zemojtel T, Abedini SS, Chen W, Hosseini M, Behjati F, Haas S, Jamali P, Zecha A, Mohseni M, Puttmann L, Vahid LN, Jensen C, Moheb LA, et al. Deep sequencing reveals 50 novel genes for recessive cognitive disorders. Nature. 2011; 478:57-63.

38. Tai CY, Mysore SP, Chiu C and Schuman EM. Activityregulated N-cadherin endocytosis. Neuron. 2007; 54:771785.

39. Niethammer M, Smith DS, Ayala R, Peng J, Ko J, Lee MS, Morabito M and Tsai LH. NUDEL is a novel Cdk5 substrate that associates with LIS1 and cytoplasmic dynein. Neuron. 2000; 28:697-711.

40. Cho KO, Hunt CA and Kennedy MB. The rat brain postsynaptic density fraction contains a homolog of the Drosophila discs-large tumor suppressor protein. Neuron. 1992; 9:929-942. 